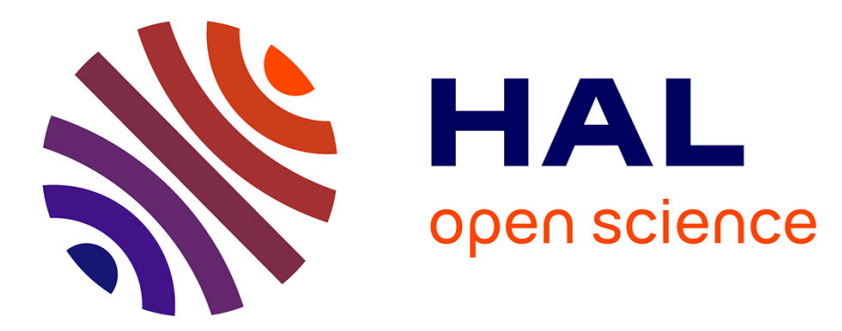

\title{
Inductance, répartition des courants et champ magnétique pour un ensemble de lignes supraconductrices
}

J. Chilo, C. Monllor

\section{- To cite this version:}

J. Chilo, C. Monllor. Inductance, répartition des courants et champ magnétique pour un ensemble de lignes supraconductrices. Revue de Physique Appliquée, 1982, 17 (8), pp.465-472. 10.1051/rphysap:01982001708046500 . jpa-00245023

\section{HAL Id: jpa-00245023 https://hal.science/jpa-00245023}

Submitted on 1 Jan 1982

HAL is a multi-disciplinary open access archive for the deposit and dissemination of scientific research documents, whether they are published or not. The documents may come from teaching and research institutions in France or abroad, or from public or private research centers.
L'archive ouverte pluridisciplinaire HAL, est destinée au dépôt et à la diffusion de documents scientifiques de niveau recherche, publiés ou non, émanant des établissements d'enseignement et de recherche français ou étrangers, des laboratoires publics ou privés. 
Classification

Physics Abstracts

$02.60-02.70-74.70-74.90$

\title{
Inductance, répartition des courants et champ magnétique pour un ensemble de lignes supraconductrices
}

\author{
J. Chilo et C. Monllor \\ Laboratoire d'Electromagnétisme ENSERG, 23, rue des Martyrs, 38031 Grenoble, France
}

(Reçu le 22 février 1982, révisé le 3 mai 1982, accepté le 7 mai 1982)

\begin{abstract}
Résumé. - Nous déterminons l'énergie libre d'un ensemble de lignes supraconductrices et la minimisons par une méthode variationnelle. Nous calculons la distribution des courants, la structure du champ magnétique et la matrice inductance du système. Les résultats obtenus pour une ligne micro-ruban supraconductrice mettent en évidence les effets de bords liés à la géométrie utilisée (ruban de largeur $2,5 \mu \mathrm{m}$ ). Ces effets peuvent être à l'origine de couplages parasites dont le rôle doit être pris en compte dans la conception de circuits intégrés réalisés en technologie Josephson. Nous montrons également l'influence des profondeurs de London sur la distribution des courants dans les supraconducteurs et sur la structure du champ magnétique en tous points de la ligne.
\end{abstract}

\begin{abstract}
We determine the free energy of a superconducting network and minimize it by a variational method. We calculate the current density distribution, the structure of the magnetic field and the inductance matrix. The results obtained for a superconducting microstrip line show the edge effects related to the geometry used (width of the strip : $2.5 \mu \mathrm{m}$ ). These effects produce « cross-talk " which must be taken into account in integrated Josephson circuit conception. We also show the influence of the London penetration depth on the distribution of the current density in superconductors and on the magnetic field structure in any point of the microstrip line.
\end{abstract}

1. Introduction. - La commutation d'une jonction Josephson se produit lorsque le courant qui la traverse devient supérieur à son courant critique. Dans les dispositifs logiques rapides à effet Josephson la commande se fait à l'aide de lignes microruban supraconductrices couplées magnétiquement au circuit. Ces lignes ont pour but de créer un champ magnétique au niveau de la jonction (diminution du courant critique [1]) ou d'induire un courant supplémentaire dans une boucle de commande (dispositifs interférométriques) [2], [3].

Dans tous les dispositifs multicommandés il est nécessaire de connaitre le champ créé par l'ensemble des lignes et la distribution des courants dans chaque ligne. Le couplage magnétique des lignes entre elles et sur le circuit se traduit par la matrice inductance.

En 1979, Alsop et al. [4] ont proposé une méthode de différences finies permettant de déterminer la configuration du champ magnétique pour un ensemble de lignes supraconductrices sur plan de masse. Leur méthode suppose qu'il n'y a pas de pénétration dans le plan de masse et nécessite l'introduction de frontières autour du système étudié; elle n'est pas très bien adaptée aux structures illimitées.

La méthode que nous présentons consiste à minimiser l'énergie libre d'un système de $N$ lignes supra- conductrices sur un plan de masse. Nous calculons ainsi la matrice inductance, les profils des densités de courant dans les supraconducteurs et la structure du champ magnétique en tous points. Nous pouvons considérer des supraconducteurs de nature différente, choisir l'un quelconque des conducteurs comme référence et il n'est pas nécessaire d'introduire des frontières artificielles. Nous supposons les lignes de longueurs infinies et donnons les résultats obtenus pour une ligne microruban.

2. Etude de l'énergie libre. - Considérons l'ensemble de $N$ lignes supraconductrices sur un plan de masse représenté sur la figure 1 . Tous les conducteurs sont supposés de longueurs infinies.

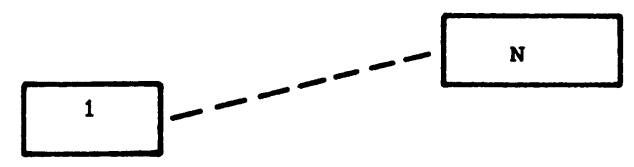

$\mathbf{N}+1$

Fig. 1. - Ensemble de lignes supraconductrices couplées.

[Coupled superconductor lines network.] 
L'énergie libre du système de lignes supraconductrices est la somme de l'énergie magnétostatique $W_{\mathrm{m}}$ et de l'énergie électrocinétique $W_{\mathrm{e}}$ des électrons supraconducteurs [5].

$$
W_{\mathrm{m}}=\frac{\mu_{0}}{2} \int_{\text {espace }} H^{2} \mathrm{~d} v
$$

$$
W_{\mathrm{e}}=\frac{\mu_{0}}{2} \int_{\text {supra }} \lambda^{2} J^{2} \mathrm{~d} v .
$$

L'énergie $W_{\mathrm{m}}$ peut s'exprimer à partir de la densité de courant et de la fonction de Green [6]. L'énergie libre, par unité de longueur du système de lignes de la figure 1 peut alors se mettre sous la forme :

$$
W=\frac{1}{2} \sum_{i=1}^{N+1} \sum_{j=1}^{N+1} \int_{S_{i}} \int_{S_{j}} J_{i}\left(r_{i}\right) J_{j}\left(r_{j}\right) G\left(r_{i}, r_{j}\right) \mathrm{d} s_{i} \mathrm{~d} s_{j}+\frac{\mu_{0}}{2} \sum_{i=1}^{N+1} \int_{S_{i}} \lambda_{i}^{2} J_{i}^{2}\left(r_{i}\right) \mathrm{d} s_{i}
$$

$J$ : densité de courant au point $r$,

$\lambda$ : profondeur de London,

$G\left(r_{i}, r_{j}\right)=-\frac{\mu_{0}}{2 \pi} \operatorname{Ln}\left|r_{j}-r_{i}\right|:$ fonction de Green en deux dimensions.

La relation (3) est une formulation variationnelle des densités de courant [7]. Nous minimiserons cette expression par la méthode des multiplicateurs de Lagrange [8].

Chaque conducteur $j$ de surface $S_{j}$ est divisé en $M_{j}$ sections élémentaires de surface $s_{i}$ dans lesquelles la densité de courant est supposée constante (Fig. 2).

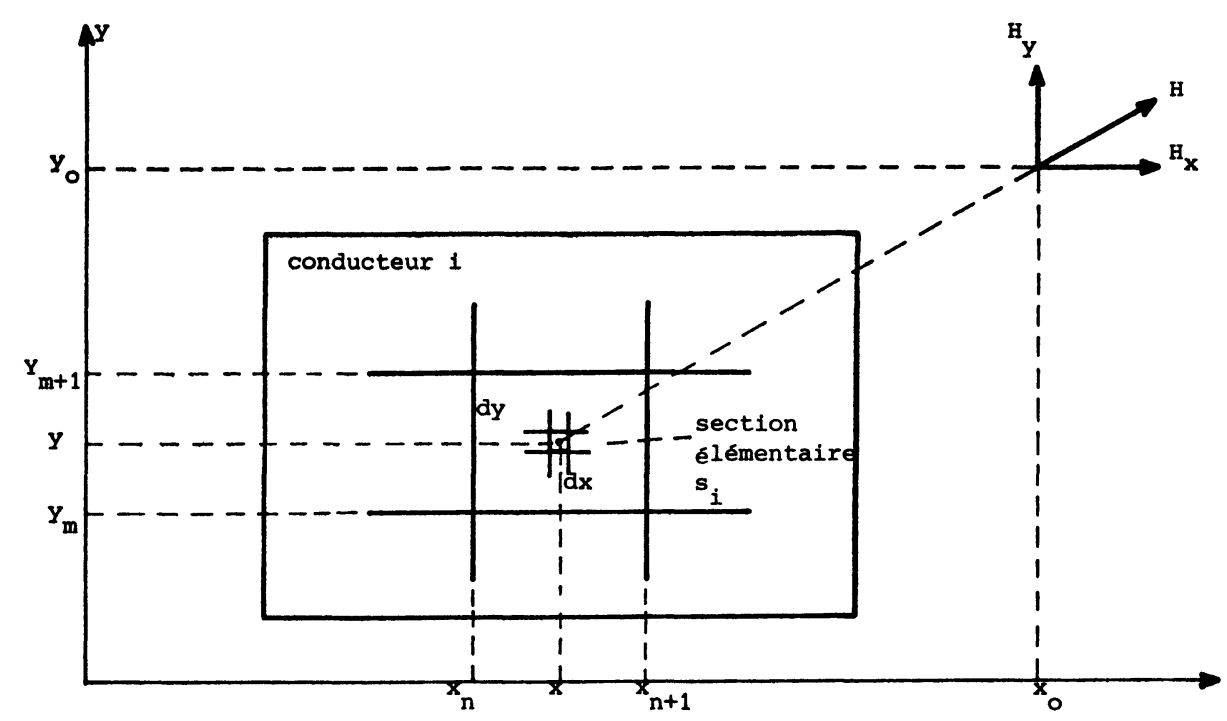

Fig. 2. - Partition élémentaire des conducteurs.

[Elementary subdivision of the conductors.]

Pour le conducteur $j$ le courant total s'écrit :

$$
I_{j}=\sum_{i=1}^{M_{i}} s_{i} J_{i}
$$

cette relation détermine la contrainte nécessaire pour construire la fonction $f$ telle que :

$$
f=\frac{1}{2} \sum_{k=1}^{M} \sum_{l=1}^{M} G_{k l} J_{k} J_{l}+\frac{\mu_{0}}{2} \sum_{k=1}^{M} s_{k} \lambda_{k}^{2} J_{k}^{2}+\sum_{j=1}^{N+1} \alpha_{j}\left[\sum_{i=1}^{M_{j}} s_{i} J_{i}-I_{j}\right]
$$

avec : $M=\sum_{j=1}^{N+1} M_{j}:$ nombre total de sections élémentaires.

$\alpha_{j}$ représentent les multiplicateurs de Lagrange.

$$
G_{k l}=-\frac{\mu_{0}}{4 \pi} \int_{x_{k}}^{x_{k+1}} \mathrm{~d} x \int_{y_{k}}^{y_{k+1}} \mathrm{~d} y \int_{x_{l}}^{x_{l}+1} \mathrm{~d} x^{\prime} \int_{y_{l}}^{y_{l+1}} \mathrm{~d} y^{\prime} \operatorname{Ln}\left[\left(x-x^{\prime}\right)^{2}+\left(y-y^{\prime}\right)^{2}\right] .
$$


Les coefficients de Green $G_{k l}$ peuvent être calculés analytiquement. Le calcul donne :

$$
\begin{aligned}
G_{k l}=G_{l k}=\frac{\mu_{0}}{96 \pi}\left[\left[\left[\left[\left\{25 u^{2} v^{2}\right.\right.\right.\right.\right. & +\left(u^{4}-6 u^{2} v^{2}+v^{4}\right) \operatorname{Ln}\left(u^{2}+v^{2}\right) \\
& \left.\left.\left.\left.\left.-8 u v\left(u^{2} \operatorname{Arctg} \frac{v}{u}+v^{2} \operatorname{Arctg} \frac{u}{v}\right)\right\}\right]_{x=x_{k}}^{x=x_{k+1}}\right]_{y=y_{k}}^{y=y_{k+1}}\right]_{x^{\prime}=x_{l}}^{x^{\prime}=x_{l+1}}\right]_{y^{\prime}=y_{l}}^{y^{\prime}=y_{l+1}}
\end{aligned}
$$

avec : $u=x-x^{\prime}, v=y-y^{\prime}$.

Le minimum de la fonction $f$ est déterminé par les relations suivantes :

$$
\begin{array}{rlrl}
\sum_{l=1}^{M} J_{l} & =0 & \\
\frac{\partial f}{\partial J_{k}} & =0 & & \text { pour } k=1,2, \ldots, M-1 \\
\frac{\partial f}{\partial \alpha_{k}} & =0 & & \text { pour } k=1,2, \ldots, N+1 .
\end{array}
$$

On obtient ainsi un système de $M-1$ équations que l'on peut écrire sous la forme matricielle suivante :

$$
\left[G_{\mathrm{p}}\right] \cdot[J]=[A] .
$$

$\left[G_{\mathrm{p}}\right]$ est la matrice carrée d'ordre $M-1$ dont les coefficients s'obtiennent à partir des coefficients de Green pondérés :

$$
G_{\mathrm{p}}(k, l)=G_{\mathrm{p}}(l, k)=\frac{1}{S}\left[G_{M M}+G_{k l}-G_{k M}-G_{l M}\right]+\mu_{0}\left(\lambda_{M}^{2}+\lambda_{k}^{2} \delta_{k l}\right)
$$

avec :

$$
\begin{array}{ll}
\delta_{k l}=1 & \text { pour } k=l \\
\delta_{k l}=0 & \text { pour } k \neq l
\end{array}
$$

Pour le calcul de $G_{\mathrm{p}}$ nous avons supposé que chaque section élémentaire de conducteur avait la même surface $s$.

$[J]$ est une matrice colonne d'ordre $M-1$ représentant les densités de courant.

[A] est une matrice colonne d'ordre $M-1$ traduisant les contraintes $\alpha_{k}$.

La matrice $[A]$ s'écrit sous la forme :

$\left.[A]=\left[\begin{array}{c}\alpha_{N+1}-\alpha_{1} \\ \vdots \\ \alpha_{N+1}-\alpha_{1} \\ \hdashline \vdots \\ \hdashline \alpha_{N+1}-\alpha_{i}^{-} \\ \vdots \\ \alpha_{N+1}-\alpha_{i} \\ \hdashline \vdots \\ \overline{\alpha_{N+1}-\alpha_{N}} \\ \vdots \\ \alpha_{N+1}-\alpha_{N} \\ 0 \\ \vdots \\ 0\end{array}\right]\right\} M_{i}$ lignes

Pour calculer $[J]$ il faut connaitre la matrice contrainte $[A]$ dont les coefficients dépendent des conditions d'excitation des lignes. La matrice des courants $[I]$ qui traduit les contraintes réelles peut être reliée à $[A]$ par une matrice particulière qui est la matrice inductance $[L]$.

3. Matrice inductance et densités de courant. L'énergie libre du système peut s'exprimer à partir des coefficients d'induction mutuelle $L_{i j}$ entre conducteurs $i$ et $j$ sous la forme :

$$
W=\frac{1}{2} \sum_{i=1}^{N} \sum_{j=1}^{N} L_{i j} I_{i} I_{j} .
$$

Dans cette expression $L_{i i}$ représente le coefficient d'induction propre du conducteur $i$.

Pour minimiser cette énergie nous construisons une fonction $g$ à partir des contraintes définies dans la relation (4). Le minimum de $g$ est obtenu par les relations :

$$
\begin{gathered}
\sum_{i=1}^{N+1} I_{l}=0 \\
\frac{\partial g}{\partial I_{j}}=0 \text { pour } j=1,2 \ldots, N \\
\frac{\partial g}{\partial \alpha_{i}}=0 \text { pour } i=1,2 \ldots, N .
\end{gathered}
$$


On obtient ainsi un système de $N$ équations qui s'exprime sous la forme matricielle suivante :

$$
[L] \cdot[I]=[a]
$$

avec :

$$
[a]=\left[\begin{array}{c}
\alpha_{N+1}-\alpha_{1} \\
\alpha_{N+1}-\alpha_{2} \\
\vdots \\
\alpha_{N+1}-\alpha_{N}
\end{array}\right]
$$

La relation (9) permet d'écrire :

$$
[J]=\left[G_{\mathrm{p}}\right]^{-1} \cdot[A]=[Q] \cdot[A] .
$$

Le coefficient $J_{j}$ de la matrice [ $\left.J\right]$ est donné par la relation :

$$
J_{j}=\sum_{k=1}^{M-1} Q_{j k} A_{k}
$$

Pour le conducteur $i$ le courant total s'écrit :

$$
I_{i}=s \sum_{j=M_{i-1}+1}^{M_{i-1}+M_{i}} J_{j}=s \sum_{j=M_{i-1}+1}^{M_{i-1}+M_{i}} \sum_{k=1}^{M-1} Q_{j k} A_{k} .
$$

Dans cette relation la sommation porte sur les densités de courant comprises entre $M_{i-1}+1$ et $M_{i-1}+M_{i}$. On remarque que le terme $A_{k}$ est constant et qu'il a pour valeur :

$$
A_{k}=\alpha_{N+1}-\alpha_{k} \text { pour } \quad M_{i-1}+1 \leqslant k \leqslant M_{i-1}+M_{i} .
$$

Compte tenu de la relation (15) on a :

$$
A_{k}=a_{i} \text { pour } M_{i-1}+1 \leqslant k \leqslant M_{i-1}+M_{i} .
$$

Pour l'ensemble des $N$ conducteurs le courant peut s'écrire :

$$
[I]=[P] \cdot[a]
$$

avec :

$$
P_{i j}=s \sum_{m=M_{i-1}+1}^{M_{i-1}+M_{i}} \sum_{n=M_{j-1}+1}^{M_{j-1}+M_{j}} Q_{m n}
$$

Compte tenu de la relation (14) la matrice inductance s'obtient par inversion de la matrice $[P]$ :

$$
[L]=[P]^{-1} \text {. }
$$

Lorsque l'on fixe les conditions d'excitation de chaque ligne, la matrice courant $[I]$ est connue et les densités de courant dans les conducteurs s'obtiennent à partir des relations (21), (20) et (16).

4. Champ magnétique. - Un élément de surface $\mathrm{d} x \mathrm{~d} y$ de densité de courant $J(x, y)$ produit en un point $\left(x_{0}, y_{0}\right)$ un champ magnétique que l'on décompose suivant $\mathrm{O} x$ et $\mathrm{O} y$. Pour une section élémentaire $i$ représentée sur la figure 2 ces composantes de champ se calculent par le théorème d'Ampère :

$$
\begin{aligned}
& H_{x_{i}}=-\frac{1}{2 \pi} \int_{x_{n}}^{x_{n+1}} \int_{y_{m}}^{y_{m+1}} \frac{\left(y_{0}-y\right) J(x, y) \mathrm{d} x \mathrm{~d} y}{\left(x_{0}-x\right)^{2}+\left(y_{0}-y\right)^{2}} \\
& H_{y_{i}}=\frac{1}{2 \pi} \int_{x_{n}}^{x_{n+1}} \int_{y_{m}}^{y_{m+1}} \frac{\left(x_{0}-x\right) J(x, y) \mathrm{d} x \mathrm{~d} y}{\left(x_{0}-x\right)^{2}+\left(y_{0}-y\right)^{2}} .
\end{aligned}
$$

Les densités de courant dans une section $i$ sont supposées constantes. On peut donc exprimer le champ total créé par l'ensemble des sous-sections de tous les conducteurs sous la forme :

$$
\begin{aligned}
& H_{x}=-\sum_{i=1}^{M} G_{x_{i}} J_{i} \\
& H_{y}=\sum_{i=1}^{M} G_{y_{i}} J_{i} .
\end{aligned}
$$

$G_{x}$ et $G_{y}$ peuvent être calculés analytiquement. Le calcul donne :

$$
\begin{aligned}
& G_{x}=\frac{1}{4 \pi}\left[\left[2 v \operatorname{Arctg} \frac{u}{v}+u \operatorname{Ln}\left(u^{2}+v^{2}\right)\right]_{u_{1}}^{u_{2}}\right]_{v_{1}}^{v_{2}} \\
& G_{y}=\frac{1}{4 \pi}\left[\left[2 u \operatorname{Arctg} \frac{v}{u}+v \operatorname{Ln}\left(u^{2}+v^{2}\right)\right]_{u_{1}}^{u_{2}}\right]_{v_{1}}^{v_{2}}
\end{aligned}
$$

avec :

$$
\begin{array}{ll}
u_{2}=x_{0}-x_{n+1} & u_{1}=x_{0}-x_{n} \\
v_{2}=y_{0}-y_{m+1} & v_{1}=y_{0}-y_{m} .
\end{array}
$$

A partir des relations (26) et (27) et compte tenu des densités de courant déterminées dans le paragraphe précédent on peut calculer en chaque point les composantes $H_{x}$ et $H_{y}$ du champ magnétique.

5. Résultats. - La géométrie que nous avons étudiée est représentée sur la figure 3 . Le conducteur de référence est en niobium $(\lambda=860 \AA)$ et le ruban en plomb $(\lambda=1370 \AA)$. Les dimensions de la ligne précisées sur la figure 3 correspondent sensiblement à celles utilisées pour une technologie de $2,5 \mu \mathrm{m}$.

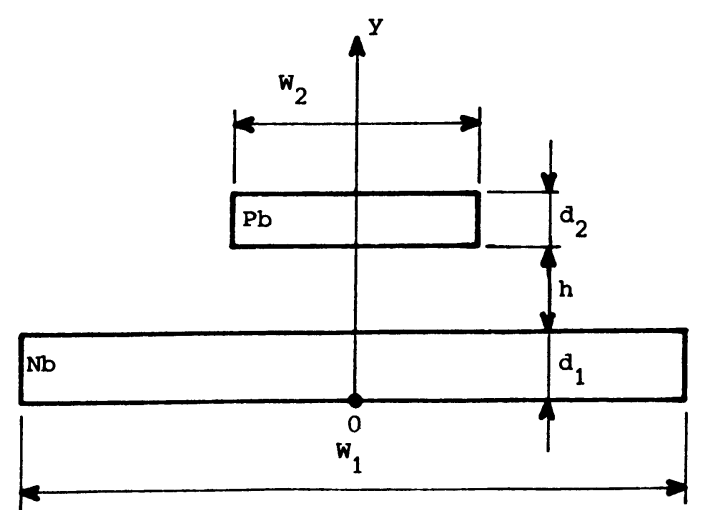

Fig. 3. - Géométrie de la ligne. $W_{1}=5 \mu \mathrm{m}, d_{1}=0,33 \mu \mathrm{m}$, $h=0,1775 \mu \mathrm{m}, W_{2}=2,5 \mu \mathrm{m}, d_{2}=0,22 \mu \mathrm{m}$.

[Line geometry.] 
5.1 Calcul de L'inductance. - Pour tester la convergence de la méthode utilisée nous avons calculé l'inductance $L$ de la ligne pour différentes partitions $M$ des conducteurs. Nous avons obtenu :

$$
\begin{array}{ll}
M=16 \text { sections } & L=0,1683 \mu \mathrm{H} / \mathrm{m} \\
M=64 \text { sections } & L=0,1646 \mu \mathrm{H} / \mathrm{m} \\
M=256 \text { sections } & L=0,1631 \mu \mathrm{H} / \mathrm{m} .
\end{array}
$$

Ces résultats montrent qu'une bonne valeur approchée de l'inductance peut être obtenue avec une faible partition. Dans ce cas les densités de courant seront entachées d'erreur. Toutefois les contraintes définies dans la relation (4) entraînent une compensation globale des erreurs dans le calcul de l'inductance.

Nous avons vérifié le bon accord de nos résultats avec ceux de Chang [9] qui prend en compte les effets de bords à l'aide d'une transformation conforme.

5.2 Densités De Courant. - La densité de courant étant une grandeur ponctuelle, sa détermination nécessite l'utilisation de la partition la plus grande possible. Les résultats que nous présentons dans ce qui suit ont été obtenus en découpant les conducteurs en sections élémentaires de dimensions : $1562,5 \AA$ et $550 \AA$ suivant les directions $\mathrm{O} x$ et $\mathrm{O} y$ respectivement. Ces valeurs conduisent à une matrice carrée $\left[G_{\mathrm{p}}\right]$ d'ordre 256. Le courant continu total traversant la ligne supraconductrice a été fixé à $1 \mathrm{~mA}$.

5.2.1 Densité de courant dans le ruban. - $\mathrm{La}$ figure 4 représente les variations de la densité de courant $J$ dans le ruban lorsque l'on se déplace d'un bord à l'autre du ruban parallèlement à l'axe $O x$.

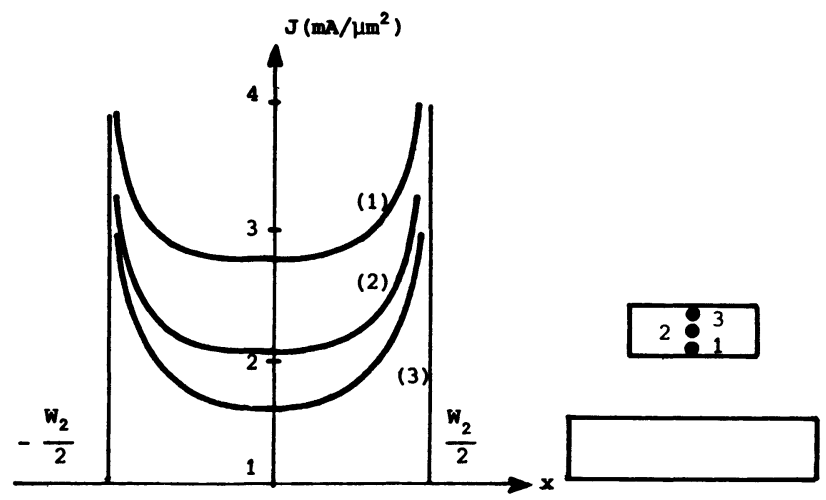

Fig. 4. - Densité de courant dans le ruban $(I=1 \mathrm{~mA})^{\circ}$ : (1) : Près du plan inférieur : $y=0,535 \mu \mathrm{m}$. (2) : Au voisinage du milieu : $y=0,590 \mu \mathrm{m}$. (3) : Près du plan supérieur : $y=0,700 \mu \mathrm{m}$.

[Current density in the strip $(I=1 \mathrm{~mA}):(1):$ Near the lower plane : $y=0.535 \mu \mathrm{m}$. (2) : Near the middle : $y=0.590 \mu \mathrm{m}$. (3) : Near the upper plane : $y=0.700 \mu \mathrm{m}$.]

Cette famille de courbes montre l'influence des bords du ruban et du conducteur de référence. Dans les différentes sections élémentaires du ruban les courants circulent dans le même sens; il en résulte une accumulation des lignes de courant sur les bords et une densité plus importante.

Sur la figure 5 la courbe 1 représente les variations de $J$, près de la face supérieure du ruban, calculées d'après la méthode énergétique. La courbe 2 a été tracée à partir de la relation :

$$
J(x)=\frac{J(0, y)}{\sqrt{1-\left(\frac{x}{W / 2}\right)^{2}}} .
$$

Cette relation est valable pour des rubans d'épaisseur nulle et se justifie toutefois dans les conducteurs normaux de faibles épaisseurs. Elle est mal vérifiée dans les supraconducteurs car la profondeur de London limite la pénétration du courant et accentue les effets de bords. Nous rappelons que $J$ représente la densité volumique de courant dont la valeur n'est pas nulle sur la surface intérieure du ruban.

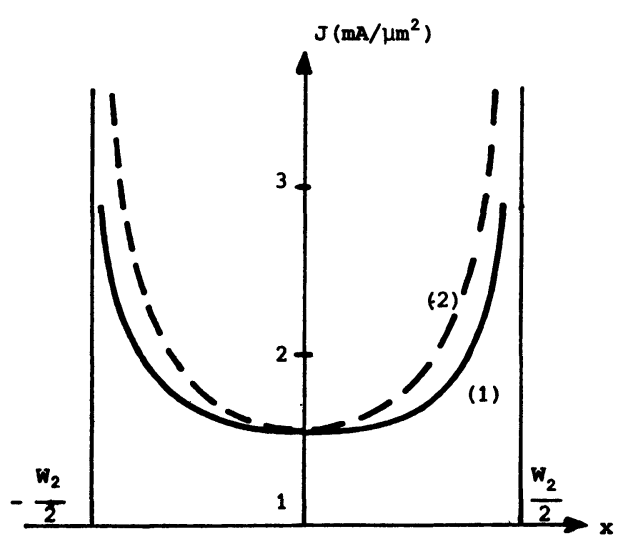

Fig. 5. - Densité de courant près du plan supérieur du ruban. $y=0,700 \mu \mathrm{m}, I=1 \mathrm{~mA}$. (1) : Méthode énergétique. (2) : Expression analytique (relation 30 ).

[Current density near the upper plane of the strip. $y=0.700 \mu \mathrm{m}, I=1 \mathrm{~mA}$. (1) : Energy method. (2) : Analytical expression (relation 30$)$.]

La figure 6 représente les densités de courant dans le ruban lorsque l'on se déplace suivant son axe de symétrie $O y$. La courbe 1 est relative aux résultats obtenus par notre méthode et la courbe 2 a été tracée en supposant une variation exponentielle de la densité de courant du type :

$$
J(y)=J_{0} \exp \left(-\frac{y}{\lambda}\right)
$$

$J_{0}$ représente la densité de courant volumique en surface. Sa valeur peut être déterminée en supposant qu'il n'y a pas d'effets de bords. Pour un courant de $1 \mathrm{~mA}, J_{0}=3,65 \mathrm{~mA} / \mu \mathrm{m}^{2}$. La différence entre les courbes 1 et 2 est due au fait que la profondeur de pénétration dans le ruban $\left(\lambda_{2}=1370 \AA\right)$ est comparable à l'épaisseur du ruban $\left(d_{2}=2200 \AA\right)$. Dans 


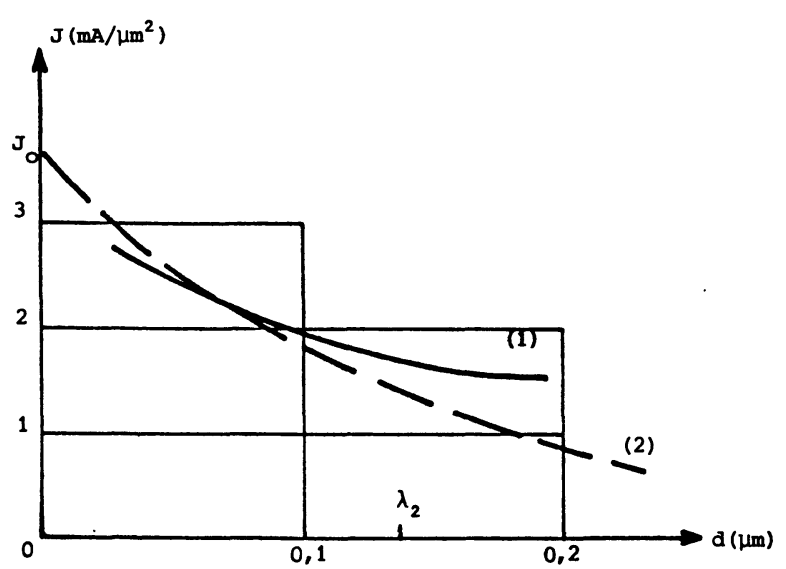

Fig. 6. - Densité de courant sur l'axe de symétrie du ruban. $x=0, I=1 \mathrm{~mA}$. (1) : Méthode énergétique. (2) : Variation exponentielle (relation 31). La distance $d$ est mesurée à partir du plan inférieur du ruban.

[Current density on the axis of symmetry of the strip. $x=0$, $I=1 \mathrm{~mA}$. (1) : Energy method. (2) : Exponential variation (relation 31). Distance $d$ is measured from the lower plane of the strip.]

ces conditions la relation approchée (31) n'est pas valable. Cette différence est accentuée par la présence du conducteur de référence parcouru par un courant de sens opposé à celui du ruban. Ceci entraîne une augmentation des densités de courant sur les faces en regard des deux supraconducteurs au détriment des faces extérieures.

5.2.2 Densités de courant dans le conducteur de référence. - La figure 7 représente les variations de cette densité lorsque l'on se déplace d'un bord à l'autre du conducteur de référence parallèlement au ruban. Comme précédemment la présence du ruban conduit à une densité maximale sur la face supérieure et à une concentration importante des courants sur la portion du conducteur de référence directement en regard du ruban. On note aussi des effets de bords prononcés qui peuvent être à l'origine de couplages parasites entre des circuits dont les plans de masse sont rapprochés.

La figure 8 montre les variations de la densité de courant dans le plan de référence suivant $\mathrm{O} y$ et la comparaison des résultats avec une variation semblable à celle donnée par la relation (31) avec :

$$
J_{0}=4,54 \mathrm{~mA} / \mu \mathrm{m}^{2} .
$$

La profondeur de London dans le niobium $\left(\lambda_{1}=860 \AA\right)$ étant faible devant l'épaisseur du conducteur de référence $\left(d_{1}=3300 \AA\right)$ les deux courbes tracées figure 8 sont pratiquement confondues.

5.3 ChAMP MAGNÉTIQUE. - Le champ magnétique $H$ résulte de la contribution de l'ensemble des densités de courant. Pour les mêmes raisons que pour le calcul de l'inductance, la détermination de $H$ ne requiert pas nécessairement une partition très importante.

5.3.1. Composante du champ magnétique parallèle à la ligne. - La figure 9 représente les variations de la composante $H_{x}$ du champ magnétique dans des plans parallèles à la ligne.

- Dans le conducteur de référence $\lambda_{1}<d_{1}$ et l'hypothèse classique consistant à considérer la composante du champ $H_{x}$ nulle est bien vérifiée. Ceci n'est pas vrai au-dessus du ruban et la composante $H_{x}$ non nulle peut introduire des couplages parasites entre circuits.

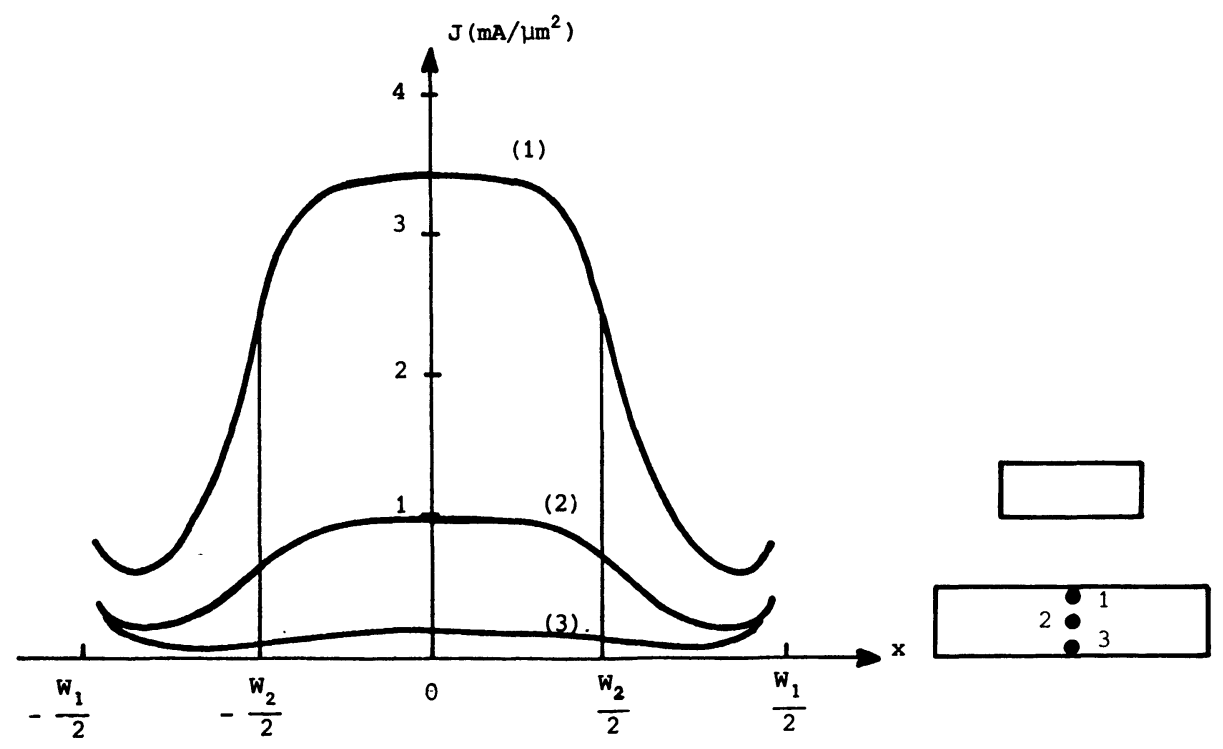

Fig. 7. - Densité de courant dans le plan de masse $(I=1 \mathrm{~mA})$. (1) : Près du plan supérieur : $y=0,3025 \mu \mathrm{m}$. (2) : Au voisinage du milieu : $y=0,1925 \mu \mathrm{m}$. (3) : Près du plan inférieur": $y=0,0275 \mu \mathrm{m}$.

[Current density in the ground plane $(I=1 \mathrm{~mA})$. (1) : Near the upper plane : $y=0.3025 \mu \mathrm{m}$. (2) : Near the middle : $y=0.1925 \mu \mathrm{m}$. (3) : Near the lower plane : $y=0.0275 \mu \mathrm{m}$.] 


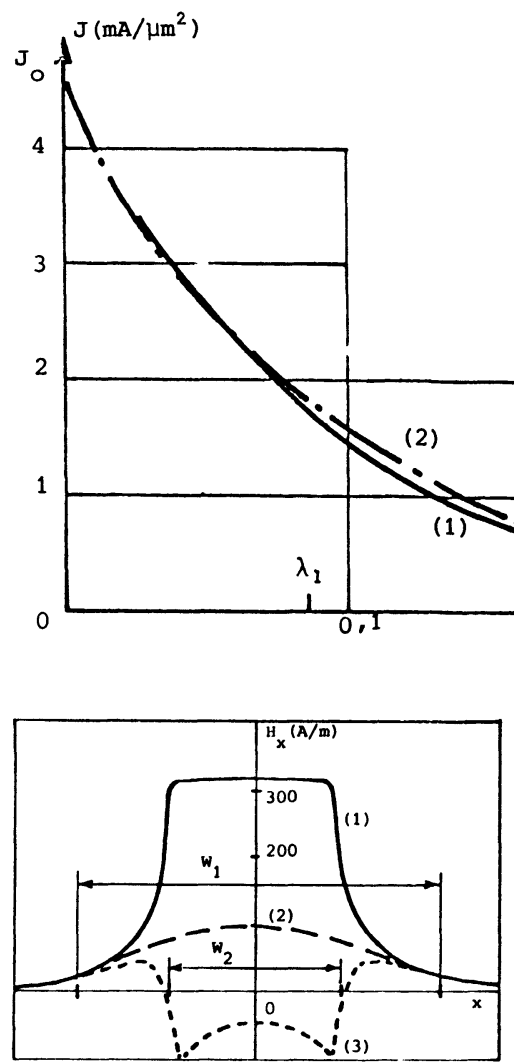

9 a. Dans le ruban

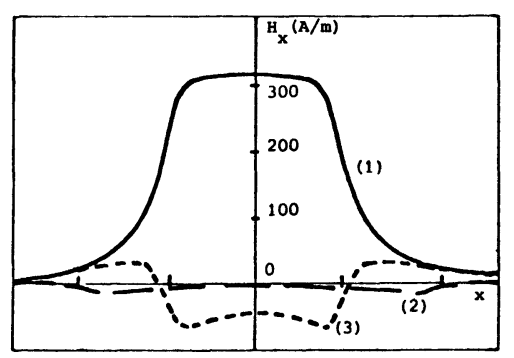

9 b. A l'extérieur de la ligne

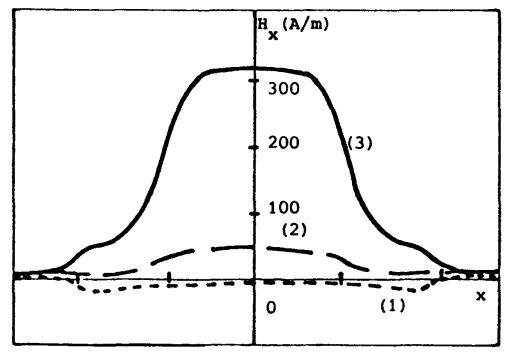

9 c. Dans le plan de masse

Fig. 9. - Composante $H_{x}$ du champ magnétique. (a) : Dans le ruban : (1) : $y=0,5075 \mu \mathrm{m},(2): y=0,6175 \mu \mathrm{m}$, (3) : $y=0,7275 \mu \mathrm{m}$. (b) : A l'extérieur de la ligne : (1) : $y=0,41875 \mu \mathrm{m},(2): y=-0,1 \mu \mathrm{m},(3): y=0,8275 \mu \mathrm{m}$.

(c) : Dans le plan de masse :(1) :y=0,(2):y=0,165 $\mu \mathrm{m}$, (3) : $y=0,33 \mu \mathrm{m}$.

[Component $H_{x}$ of the magnetic field. (a) : In the strip : (1) $: y=0.5075 \mu \mathrm{m},(2): y=0.6175 \mu \mathrm{m},(3): y=0.7275 \mu \mathrm{m}$. (b) : Out of the line :(1): $y=0.41875 \mu \mathrm{m}$, (2) :y=-0.1 $\mu \mathrm{m}$,

(3) : $y=0.8275 \mu \mathrm{m}$. (c) : In the ground plane :(1) :y=0,

(2) : $y=0.165 \mu \mathrm{m},(3): y=0.33 \mu \mathrm{m}$.]
Fig. 8. - Densité de courant sur l'axe de symétrie du plan de masse. $x=0, I=1 \mathrm{~mA}$. (1) : Méthode énergétique. (2) : Variation exponentielle (relation 31). La distance $d$ est mesurée à partir du plan supérieur du plan de masse.

[Current density on the axis of symmetry of the ground plane. $x=0, I=1 \mathrm{~mA}$. (1) : Energy method. (2) : Exponential variation (relation 31). Distance $d$ is measured from the upper plane of the ground plane.]

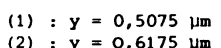
(2) : $: y=0,6175 \mu \mathrm{m}$
(3) : $y=0,7275 \mu \mathrm{m}$

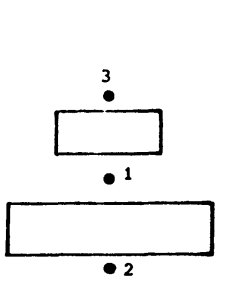

(1) : $y=0,41875 \mu \mathrm{m}$ (2): $: y=-0,1 \quad \mu \mathrm{m}$

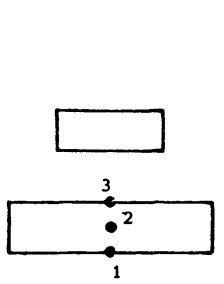

(1) : $y=0$

(2) : $y=0,165 \mu \mathrm{m}$ (3) : $: y=0,33 \mu \mathrm{m}$
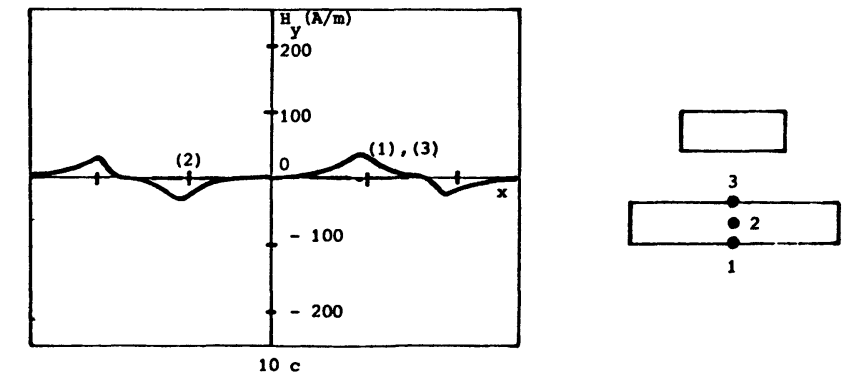

Fig. 10. - Composante $H_{y}$ du champ magnétique. Les conditions sont celles de la figure 9.

[Component $H_{y}$ of the magnetic field. The conditions are those of figure 9.]

- Dans le diélectrique la présence du ruban entraîne une forte concentration du champ magnétique; dans la zone d'influence du ruban ce champ est sensiblement homogène mais sa valeur est cependant inférieure au rapport $I / W$ (dans notre cas 
$I / W=400 \mathrm{~A} / \mathrm{m})$. Les effets de bords du ruban et du conducteur de référence se manifestent par des perturbations sur la valeur du champ $H_{x}$.

- Dans les supraconducteurs les variations de $H_{x}$ suivant l'axe $O y$ suivent celles des densités de courant. Nous avons donc une variation sensiblement exponentielle dans le plan de référence.

5.3.2 Composante du champ magnétique perpendiculaire à la ligne. - La figure 10 représente les variations de la composante $H_{y}$ dans les mêmes conditions que celles de la figure 9.

La symétrie géométrique de la ligne explique que $H_{y}$ soit nul sur l'axe $\mathrm{O} y$. Les effets de bords du ruban et $\mathrm{du}$ conducteur de référence sont à l'origine des extrema de $H_{y}$ au voisinage des bords. D'une manière générale la composante $H_{y}$ est faible devant $H_{x}$ sauf sur les bords du ruban où leurs valeurs sont comparables.

6. Conclusion. - La méthode proposée est basée sur la minimisation de l'énergie libre d'un ensemble de lignes supraconductrices en imposant, pour chacune des lignes, le courant total. Ces contraintes entraînent, dans le calcul de l'inductance et du champ magnétique, une compensation des erreurs liées à la partition des conducteurs; il n'est pas nécessaire d'utiliser un grand nombre de sections élémentaires pour déterminer ces grandeurs. Par contre le caractère ponctuel de la densité de courant implique un nombre plus important de sections.

Les résultats obtenus pour une ligne niobiumplomb en technologie $2,5 \mu \mathrm{m}$ mettent en évidence l'influence du plan de masse et des bords du ruban sur la distribution des courants. Le caractère supraconducteur des lignes rend ces effets plus importants que dans les conducteurs ordinaires.

La géométrie et les matériaux utilisés montrent que le champ magnétique au-dessus du ruban n'est pas nul. Pour éviter le rayonnement de la ligne (effet de blindage), il faudrait réduire l'épaisseur du diélectrique, augmenter l'épaisseur du ruban ou choisir des matériaux dont la profondeur de London serait plus faible.

Les programmes de calcul s'appliquent à des dispositifs plus complexes comportant plusieurs lignes sur plan de masse. La connaissance de la structure du champ magnétique créé par l'ensemble de lignes est fondamentale pour la détermination des caractéristiques de commande de circuits intégrés à effet Josephson, compte tenu de leur environnement réel.

Les réactions des lignes entre elles peuvent être calculées et minimisées pour diminuer les couplages parasites. Le couplage entre lignes sera optimisé pour réaliser des transformateurs répartis ou des coupleurs en technologie microruban.

L'ensemble des résultats obtenus à partir de considérations électromagnétiques doit permettre de déterminer des règles de conception de circuits intégrés supraconducteurs.

La méthode développée peut être transposée à des lignes conductrices normales. Les règles de conception pourraient alors s'appliquer à tout dispositif intégré, pour logique rapide ou pour micro-onde, pour lesquels le transfert des signaux s'effectue au moyen de lignes microruban.

\section{Bibliographie}

[1] Matisoo, J., Proc. IEEE 55 (1967) 172.

[2] Jaklevic, R. C., Lambe, J., Mercereau, J. E., Silver, A. H., Phys. Rev. A 140 (1965) 1628.

[3] Fulton, T. A., Superconductors Applications : SQUIDS and Machines (Plenum Press, N.Y.) 1977, Chap. IV.

[4] Alsop, L. E., Goodman, A. S., Gustavson. F. G., Miranker, W. L., J. Comput. Phys. 31 (1979) 216.
[5] London, F., Proc. R. Soc. (London) A 152 (1935) 24.

[6] Collin, R. E., Field theory of guided waves (Mc GrawHill Book Co., N.Y.) 1960, Chap. II.

[7] Chang, W. H., IEEE Trans. Mag. Mag. 17 (1981) 764

[8] Morse, P. M., Feshbach, H., Methods of theoretical Physics (Mc Graw-Hill Book Co, N.Y.) 1953.

[9] Chang, W. H., J. Appl. Phys. 50 (1979) 8129. 